\title{
Circular cladding waveguides in Pr:YAG fabricated by femtosecond laser inscription: Raman, luminescence properties and guiding performance
}

\author{
Quanxin Yang ${ }^{1}$, Hongliang Liu' ${ }^{1,2,5 *}$, Shan $\mathrm{He}^{1}$, Qingyu $\mathrm{Tian}^{4}$, Bin $\mathrm{Xu}^{4}$ and \\ Pengfei $\mathrm{Wu}^{1,3}$
}

We report on the fabrication of circular cladding waveguides with cross-section diameters of 60-120 $\mu$ m in Pr:YAG crystal by applying femtosecond laser inscription. The fabricated waveguides present 2D guidance on the cross-section and fairly low propagation losses. Multiple high-order guiding modes are observed in waveguides with different diameters. Corresponding simulation results reveal the origin of a specific kind of guiding modes. Confocal micro-Raman ( $\mu$-Raman) experiments demonstrate the modification effects in femtosecond laser affected areas and ascertain the refractive index induced guiding mechanism. In addition, luminescence emission properties of $\mathrm{Pr}^{3+}$ ions at waveguide volume are well preserved during the femtosecond laser inscription process, which may result in a potential high-power visible waveguide laser.

Keywords: femtosecond laser inscription; cladding waveguides; Pr:YAG crystal; luminescence emission

Yang QX, Liu HL, He S, Tian QY, Xu B et al. Circular cladding waveguides in Pr:YAG fabricated by femtosecond laser inscription: Raman, luminescence properties and guiding performance. Opto-Electron Adv 4, 200005 (2021).

\section{Introduction}

In the past decades, femtosecond laser inscription (FLI) as a new method for micromachining ${ }^{1-2}$, has emerged to be an effective technology for optical waveguide fabrication in various transparent dielectric materials. And in recent years, various kinds of waveguides and functional devices have been realized in some kinds of crystals, such as $\mathrm{Yb}, \mathrm{Na}: \mathrm{CaF}_{2}{ }^{3}$, diamond ${ }^{4-5}, \mathrm{Nd}: \mathrm{GdVO}_{4}{ }^{6}$, dye polymer ${ }^{7}$, Gallium Lanthanum Sulphide glass ${ }^{8}$ and $\mathrm{LiNbO}_{3}{ }^{9-11}$. Nonlinear energy transfer that occurs during the FLI process simultaneously produces localized moderation at the focal spot. The increase, decrease of refractive index or even material plasmatization can occur depending on the utilized femtosecond laser ${ }^{12}$. In addition, waveguide structure with a tubular cladding geometry (unmodified guiding region is surrounded by laser induced low-refractive-index damage tracks) gains growing attention because the guiding area of such a waveguide can still maintain the original material properties after the FLI process $^{13-19}$. Furthermore, tubular cladding waveguide shows higher stability and consistency than the stress induced dual-line and the single-line waveguides, as well as supports orthogonal polarizations in the meantime.

Praseodymium ions $\left(\mathrm{Pr}^{3+}\right)$ present extremely complex

${ }^{1}$ Institute of Modern Optics, Nankai University, Tianjin 300350, China; ${ }^{2}$ Tianjin Key Laboratory of Optoelectronic Sensor and Sensing Network Technology, Tianjin 300350, China; ${ }^{3}$ Tianjin Key Laboratory of Micro-scale Optical Information Science and Technology, Tianjin 300350, China; ${ }^{4}$ Department of Electronic Engineering, Xiamen University, Xiamen 361005, China; ${ }^{5}$ State Key Laboratory of Crystal Materials, Shandong University, Jinan 250100, China.

*Correspondence: HL Liu,E-mail: drliuhl@nankai.edu.cn

Received: 27 February 2020; Accepted: 16 April 2020; Published: 28 February 2021

200005-1 
energy level and relatively low photon energy, which make them applicable for laser transitions with different excitation wavelengths ranging from visible to infrared. During the past few years, laser generations have been reported based on different Pr-doped fluoride materials: waveguide laser in Pr:YLF' ${ }^{20}$ bulk laser in Pr:RPC ${ }^{21}$, Pr:LiLuF ${ }^{22}, \mathrm{Ho}, \operatorname{Pr}: \mathrm{LiLuF}_{4}{ }^{23}, \operatorname{Pr}: \mathrm{LiYF}_{4}{ }^{24}, \operatorname{Pr}: \mathrm{YAlO}_{3}{ }^{25}$ and Pr:ASL ${ }^{26}$. In addition, yttrium aluminum garnet $\left(\mathrm{Y}_{3} \mathrm{Al}_{5} \mathrm{O}_{12}, \mathrm{YAG}\right)$ crystal and ceramic are widely used as substrate materials of laser operating substance because of their high thermal conductivity and mechanical strength. Different rare earth ions doping endows the doped YAG samples various luminescence and laser properties $^{27-30}$.

In this paper, we exhibit the fabrication of circular cladding waveguides with low propagation losses in Pr:YAG crystal by using FLI. By investigating the guiding mode profiles and confocal $\mu$-Raman properties, localized modification effects caused by the femtosecond laser and the guiding mechanism are revealed. In addition, luminescence and guiding properties of the guiding area are also studied.

\section{Method}

\section{Waveguide fabrication}

The Pr:YAG crystal sample (praseodymium doped yttrium aluminum garnet, $\mathrm{Pr}: \mathrm{Y}_{3} \mathrm{Al}_{5} \mathrm{O}_{12}$, doped by 0.5 at. \% $\mathrm{Pr}^{3+}$ ions, obtained from Atom Optics Co. Ltd, Shanghai, China) used in this work is cut into dimensions of $10 \mathrm{~mm} \times 6 \mathrm{~mm} \times 2 \mathrm{~mm}$ with all facets optically polished. During the waveguide fabrication process, an amplified Ti:sapphire laser system (Astrella, Coherent Inc., USA) is used as laser source, which generates linearly polarized pulses (with repetition rate of $1 \mathrm{kHz}$, central wavelength of $800 \mathrm{~nm}$, pulse width of $35 \mathrm{fs}$, and single pulse energy of $7 \mathrm{~mJ}$ ). Watt Pilot motorized attenuator and a neutral density filter are utilized to adjust the laser power precisely, and specifically, single pulse energy of $0.23 \mu \mathrm{J}$ is used in this work. The laser beam is focused by a microscope objective $(40 \times, N A=0.6)$ into the substrate beneath a $10 \mathrm{~mm} \times 6 \mathrm{~mm}$ facet (the upper facet). The substrate is placed on a 6 -axes precision motorized stage and the scanning speed is set to be $0.8 \mathrm{~mm} / \mathrm{s}$.

Under the above experiment conditions, one damage track with vertical width of $15 \mu \mathrm{m}$ and horizontal width of $2.6 \mu \mathrm{m}$ can be inscribed via a single scan. Arrays of parallel damage tracks are inscribed following the designed geometries (the horizontal separation between ad- jacent tracks is $3 \mu \mathrm{m}$ ) so as to form desired cladding waveguides with different diameters, as shown in Fig. 1. All the waveguides are fabricated along the $y$-axis of the substrate (i.e., the waveguide lengths are all $10 \mathrm{~mm}$ ).

\section{Confocal $\mu$-Raman measurement and luminescence characterization}

Raman properties of the femtosecond laser induced damage tracks and the formed guiding core area are explored by a confocal $\mu$-Raman spectroscopy system (XperRam200, Nanobase, South Korea). A continuous laser beam $(532 \mathrm{~nm}, 30 \mathrm{~mW})$ is focused onto the endface by a microscope objective $(40 \times, N A=0.75)$. Part of the beam is affected by the Raman scattering effect of the sample and is then collected and transferred to a spectrometer system. With the help of a galvo scanning system, we can accurately gain the Raman properties of every region over the whole waveguide cross-section.

As for the luminescence characterization experiment process, a continuous laser beam at $400 \mathrm{~nm}$ is focused onto the end-face of the sample. And the emergent light from another end-face is collected and transferred to a spectrometer system which can provide the luminescence information of irradiated area in real time.

\section{Waveguide characterization and guiding mode simulation}

The near field intensity distributions of guiding modes are measured by an end-face coupling system. This system uses a $632.8 \mathrm{~nm}$ He-Ne laser as light source. A GlanTaylor prism and a $1 / 2$ waveplate at $632.8 \mathrm{~nm}$ are utilized to generate linear-polarized incident laser beam (i.e. TE and TM, TE: the electric field is parallel to the $x$-axis; TM: parallel to the $z$-axis). The linearly polarized beam is then coupled into one waveguide by a microscope objective $(40 \times, N A=0.65)$ and the transmission light is collected by a long working distance microscope objective $(50 \times, N A=0.55)$ from another end-face. The profile is subsequently imaged via a CCD camera (WCDUCD12-1310, DataRay, USA).

The guiding mode simulation process is accomplished by the finite element method (FEM). The geometry structures used for simulation, as shown in Fig. 1(b), are determined by referring to the micrographs and $2 \mathrm{D} \mu$-Raman mapping images, as shown in Fig. 1(c) and Fig. 3, respectively. Since the mechanical stress produced by the physical lattice expansion at damage tracks attenuates rapidly over distance and has 

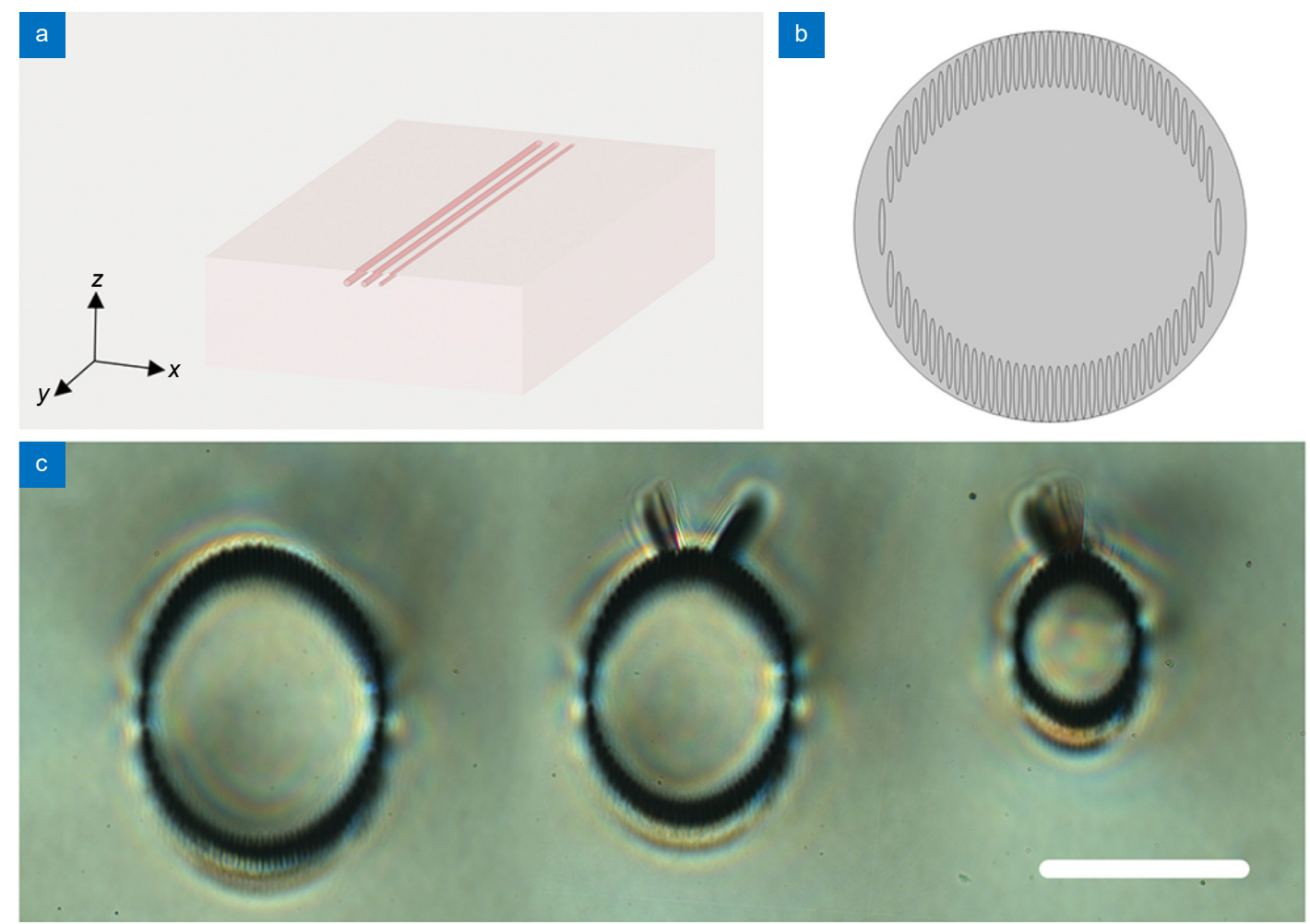

Fig. 1 | (a) The 3D schematic diagram of waveguides. Coordinate axes are defined. (b) The 2D geometry structure (corresponding to the $120 \mu \mathrm{m}$ waveguide), which is also used in the guiding mode simulation process. (c) The cross-section microscope images of the circular cladding waveguides with different diameters: $120 \mu \mathrm{m}, 100 \mu \mathrm{m}$, and $60 \mu \mathrm{m}$, respectively. The scale bar in the figure is $100 \mu \mathrm{m}$.

negligible influence on the waveguide core area, and to simplify the refractive index distribution used in the simulation, we assume a step-index configuration with the refractive index difference of -0.04 at all damage track boundaries.

\section{Results and discussion}

Figures 1(a) and 1(b) exhibit the 3D and 2D geometry structures of waveguides, and the $2 \mathrm{D}$ one is also used in the guiding mode simulation process. Figure 1 (c) shows the cross-section microscope images (optical transmission) of the circular cladding waveguides with different diameters: $120 \mu \mathrm{m}, 100 \mu \mathrm{m}$, and $60 \mu \mathrm{m}$, respectively. Since our aim of this work is to build waveguides that fit the demand of luminescence and laser generation, we are making waveguides with relatively large diameters. But according to our former experience, cladding waveguides with minimum diameter of $10 \mu \mathrm{m}$ can be fabricated in such kind of crystal. The damage tracks (of different fabricated waveguides) closest to the upper facet share a depth of $120 \mu \mathrm{m}$ beneath the upper facet. The refractive index change in the track regions is estimated to be -0.04 by measuring the NA value of the waveguides. No evident damage in the core areas of the fabricated waveguides is oberserved.

Raman properties of one fabricated waveguide with diameter of $120 \mu \mathrm{m}$ are shown in Fig. 2 and Fig. 3. Raman spectra accurately measured at different positions reveal the variation of lattice after FLI process. As we can see from Fig. 2, the Raman intensity at damage track region suffers a strong quenching without evident variation on the spectral distribution while the Raman spectra of non-processed bulk area and waveguide volume are indistinguishable.

In order to further investigate the Raman spectra variation over the whole waveguide cross-section, the $2 \mathrm{D}$ mapping is implemented and the corresponding results are presented in Fig. 3. Intensity, shift and full width at half maximum (FWHM) changes of characteristic peak $\left(259 \mathrm{~cm}^{-1}\right)$ are shown as different channels. And significant differences: Raman intensity quenching, blue shift (i.e., wavenumber decrease) and broadening of the emission peak can be observed at the damage track regions. These phenomena indicate the formation of lattice defects/damages and the elongation of bond-lengths (i.e., lattice parameter) due to the expansive stress. The partial dilatation also results in the refractive index decrease at these regions. Therefore, the refractive index induced 
guiding mechanism of such cladding waveguides can be ascertained. Otherwise, the waveguide volume manifests fairly similar Raman properties with respect to the nonprocessed bulk area, which demonstrates that the lattice structure in the guiding area is well preserved during the FLI process.

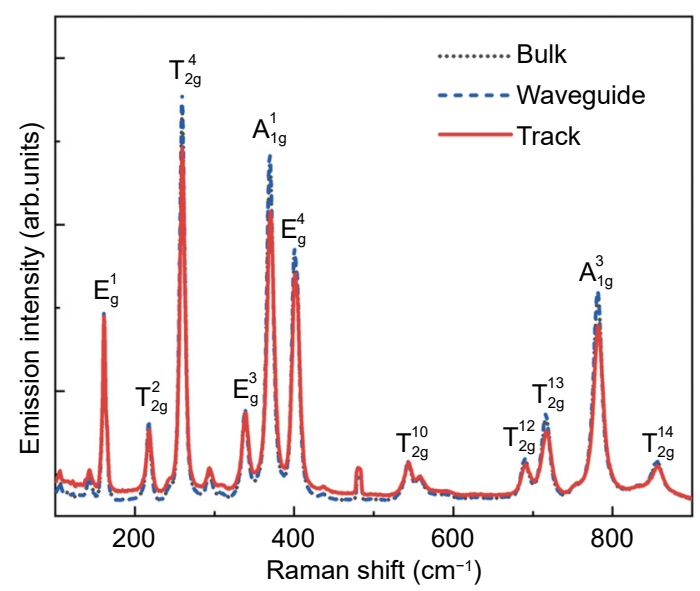

Fig. 2 | Raman spectra collected from non-processed bulk area (black dotted line, covered by almost identical blue dashed line), waveguide volume (blue dashed line), and damage track (red solid line) excited by a $532 \mathrm{~nm}$ laser. Corresponding molecular vibration modes are marked out on every peak.

A further analysis about the Raman spectra of damage tracks and the surrounding areas is introduced here. As mentioned above, significant differences are observed at the damage tracks with respect to the non-processed bulk area. Raman intensity quenching and peak broadening originates from the formation of lattice defects and damages; while for peak blue shift, the elongation of bond-lengths becomes the dominant factor. That is in good agreement with the subjective impressions, which is longer bond-lengths correspond to lower molecular vibration frequencies, closer molecular vibration energy levels, and lower Raman shift for specific peaks. But when we focus on the surrounding areas of damage tracks, a strong red shift (i.e., wavenumber increase) occurs without evident variation on Raman intensity or FWHM with respect to the non-processed bulk area, indicating a homogeneous lattice compression at these areas. In other words, regions modified by the femtosecond laser present lattice dilatation with refractive index decrease while the surrounding areas are squeezed by the modified regions, forming lattice compression with refractive index increase. This phenomenon is in agreement with several published works accomplished on different samples ${ }^{1,5}$.

Figure 4 depicts the luminescence emission spectra of the guiding volume of one fabricated $120 \mu \mathrm{m}$ waveguide and non-processed bulk area. Virtually identical emission spectra (both spectral distribution and intensity) indicate that the luminescence properties of $\mathrm{Pr}^{3+}$ ions at waveguide volume have been well preserved during the FLI process.

All the fabricated cladding waveguides support both TE and TM polarizations (following the aforesaid definition). Since the diameters of the fabricated waveguide are relatively large $(\sim 100 \mu \mathrm{m})$ compared to the incident laser wavelength $(632.8 \mathrm{~nm})$, large amounts of high-order modes are supported to propagate in the waveguides. Because of that, different modes with different intensity

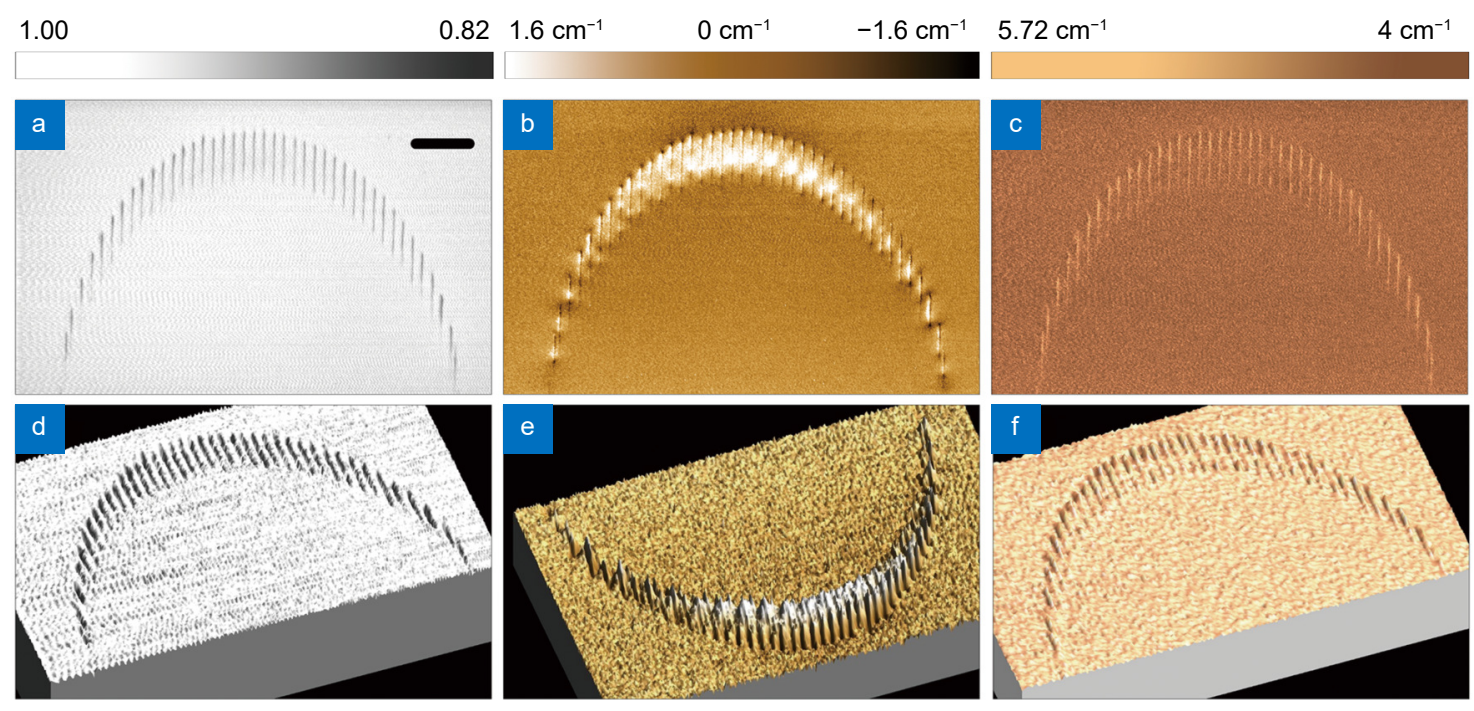

Fig. 3 | Confocal $\mu$-Raman 2D mapping results are exhibited as 2D and 3D images with imaging channels. (a) and (d) Intensity (normalized); (b) and (e) Shift; (c) and (f) FWHM of the characteristic peak at $259 \mathrm{~cm}^{-1}$, respectively. The scale bar in the figure is $20 \mu \mathrm{m}$. 
distributions and effective refractive indexes can be excited when the incident laser beam is focused on different relative positions with respect to the waveguide volume.

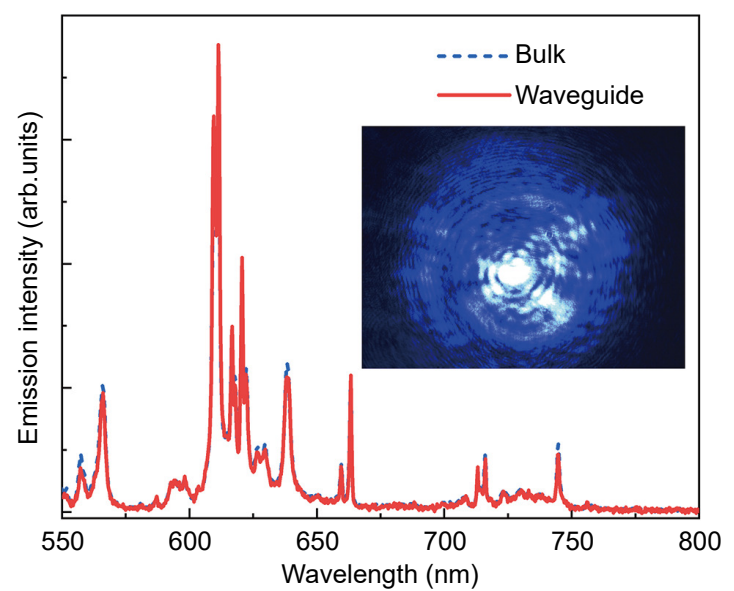

Fig. 4 | Luminescence emission spectra of waveguide volume (blue dashed line) and non-processed bulk area (red solid line). Inset shows the output end-face image captured by a color CCD camera while a laser beam at $400 \mathrm{~nm}$ has been coupled into the waveguide through another end-face.

The guiding modes with a specific kind of intensity distribution of waveguides with different diameters and the corresponding simulation results are exhibited in Fig. 5. These guiding modes possess horizontal fringes with TE polarization, which is actually the characteristic of $\mathrm{LP}_{1 \mathrm{n}}$ modes (LP mode: linearly polarized mode). It's worth mentioning that these modes only exist when the incident laser beam is focused on the positions a little bit right above or below the waveguide center. As can be seen in this figure, the experiment and the simulation results match well.

Here, we introduce a further discussion about the origin of guiding modes with this specific kind of intensity distributions. In consideration of the similarity between the observed modes and the typical modes in optical fiber, an ideal step-index circularly-symmetric fiber model that has the same refractive index profile and waveguide core area with the $60 \mu \mathrm{m}$ cladding waveguide model is established. Simulation result indicates that such fiber supports the propagation of $\mathrm{TE}_{04}$ mode, as shown in Fig. 6(a). Circularly symmetric fiber supports the propagation of modes with the same electric field distribution but different poloidal rotation angles about the fiber axis, and these modes present nearly indistinguishable effective refractive indexes, which means, they are degenerate. In addition, simulation method that we used (using effective refractive indexes to distinguish modes, mode analysis, FEM) tends to show a combined mode as result instead of several degenerate modes if they have
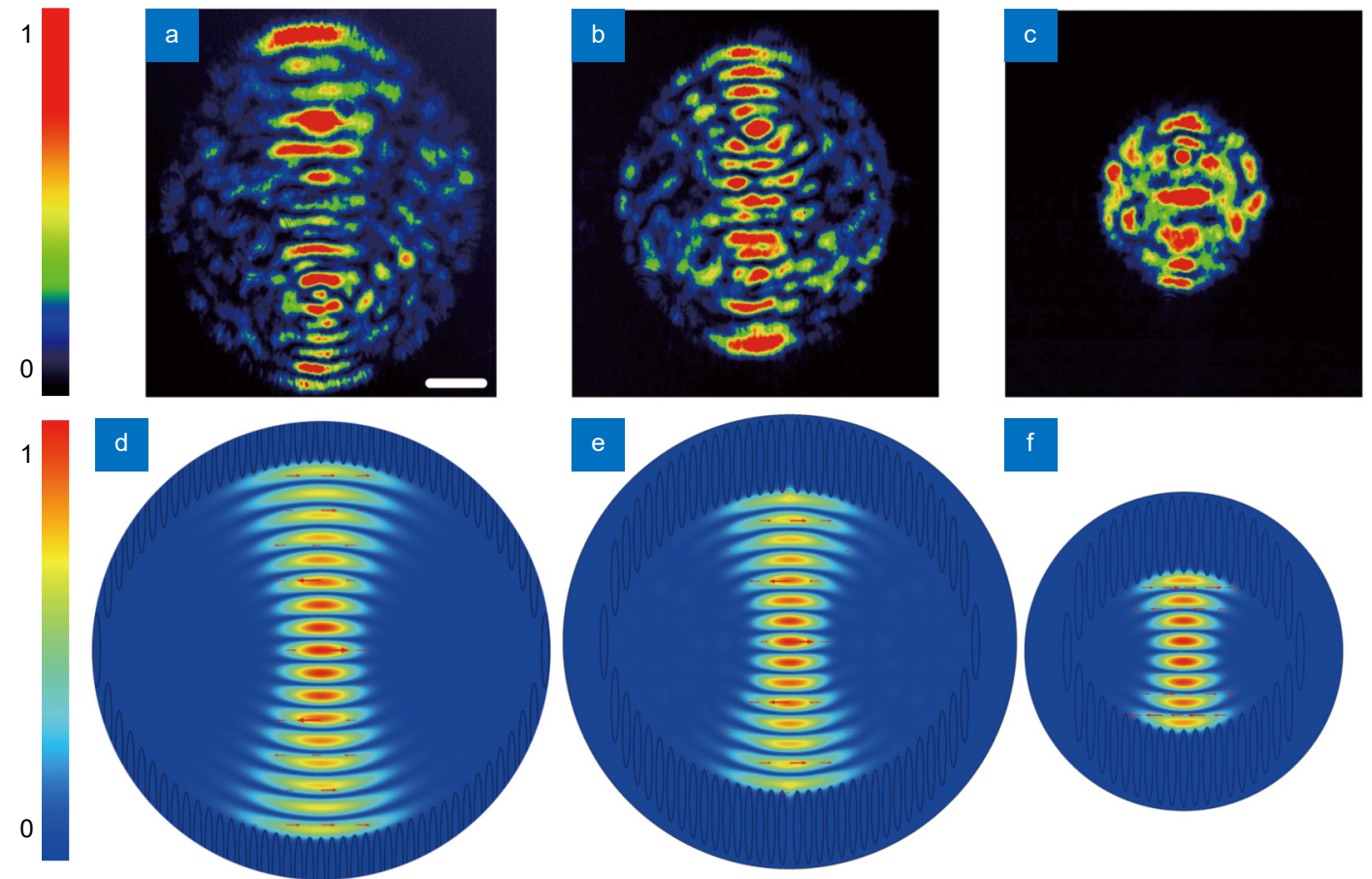

Fig. 5 | A specific kind of modes with an intensity distribution of horizontal fringes in waveguides with different diameters. (a) and (d) $120 \mu \mathrm{m}$; (b) and (e) $100 \mu \mathrm{m}$; (c) and (f) $60 \mu \mathrm{m}$. (a-c) Experimental mode intensity distributions gained by the end-face coupling system using a laser beam at $632.8 \mathrm{~nm}$. (d-f) Corresponding simulation results. The effective refractive indexes are $1.8392,1.8390$ and 1.8389 , respectively. Red arrows point to the directions of electric field. The scale bar in the figure is $20 \mu \mathrm{m}$. 
nearly the same effective refractive indexes. Based on the above points, gained $\mathrm{TE}_{04}$ mode can be separated into two orthogonally polarized $\mathrm{LP}_{14}$ modes. The obtained $\mathrm{LP}_{14}$ modes present the same electric field distribution with a poloidal rotation of $90^{\circ}$, and Fig. 6(c) shows the $\mathrm{LP}_{14}$ mode with horizontal polarization. It should be noticed that, generally, a specific LP mode is viewed as a combination of several vector modes including TE, TM, $\mathrm{EH}$, and HE because LP mode is gained using weakly guiding approximation. But since LP mode can be expressed by vector modes, vice versa, a specific vector mode can be expressed by several LP modes.

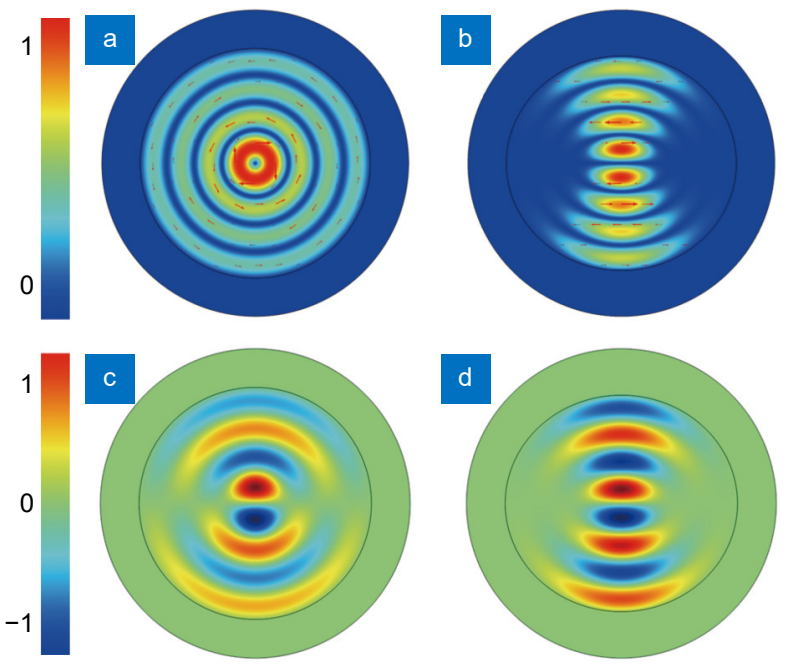

Fig. 6 | Simulated mode profiles and corresponding horizontally polarized electric field distributions of (a) and (c) the circularly symmetric fiber model; (b) and (d) the elliptical fiber model. The effective refractive indexes are 1.8395 and 1.8394, respectively. Red arrows point to the directions of electric field.

Since these $\mathrm{LP}_{14}$ modes are degenerate, they are more inclined to combine and form a circularly symmetric $\mathrm{TE}_{04}$ mode in simulation, which means, a single $\mathrm{LP}_{14}$ mode will not emerge under such condition. But if under non-circularly-symmetric condition, such as in elliptical fiber, previous degenerate modes with different polarizations will manifest similar electric-field distributions and slightly different effective refractive indexes, scilicet the removal of degeneracy ${ }^{31}$. To confirm the reliability of this thought, an elliptical fiber model with major axis of $60 \mu \mathrm{m}$ and minor axis of $56 \mu \mathrm{m}$ is established. And under such simulation condition, single $\mathrm{LP}_{14}$ modes can be observed, as shown in Figs. 6(b) and 6(d), which is coincident with the experiment and simulation results of the fabricated $60 \mu \mathrm{m}$ waveguide. This result demonstrates the non-circular symmetry of the fabricated waveguides, also suggests that with refined design, the fabric- ated waveguides can support the propagation of circularly symmetric modes. These conclusions are also applicable for the waveguides with different diameters.

The losses of the waveguides at $632.8 \mathrm{~nm}$ when the incident laser is differently polarized are given in Table 1. The total losses are directly measured by an optical power meter and the end-face coupling system used in the measurement of the intensity distributions of guiding modes while the coupling losses are roughly calculated by the BeamPROP module of Rsoft Photonics CAD Suite. The main simulation parameters are listed here: spot diameter of $10 \mu \mathrm{m}$; refractive index difference of -0.04 . Therefore, the propagation losses of the waveguides can be directly given and the error induced during the measurements should be less than $\pm 0.2 \mathrm{~dB} / \mathrm{cm}$. Since the beam diameter of $10 \mu \mathrm{m}$ is relatively small compared to the waveguide diameters of 100 and $120 \mu \mathrm{m}$, simulation results reveal the same coupling loss value of the waveguides with diameters of 100 and $120 \mu \mathrm{m}$. The propagation losses result shows such circular cladding waveguides in Pr:YAG present fairly low propagation losses at $632.8 \mathrm{~nm}$.

Table 1 | The losses of the fabricated cladding waveguides in Pr:YAG at $632.8 \mathrm{~nm}$.

\begin{tabular}{|c|c|c|c|c|}
\hline Pr:YAG at $632.8 \mathbf{n m}$. & & $60 \mu \mathrm{m}$ & $100 \mu \mathrm{m}$ & $120 \mu \mathrm{m}$ \\
\hline Total loss $(\mathrm{dB})$ & TE & 3.60 & 3.31 & 3.46 \\
\hline Coupling loss $(\mathrm{dB})$ & TM & 4.06 & 3.70 & 3.86 \\
\hline \multirow{2}{*}{ Propagation loss $(\mathrm{dB} / \mathrm{cm})$} & TE/TM & 3.47 & 3.16 & 3.16 \\
\hline & TE & 0.13 & 0.15 & 0.3 \\
\hline & TM & 0.59 & 0.54 & 0.7 \\
\hline
\end{tabular}

\section{Conclusions}

In conclusion, we have successfully fabricated circular cladding waveguides which support multi-mode propagation with orthogonal polarizations in Pr:YAG crystal by FLI. The fabricated waveguides show good guiding performance without any deterioration of the luminescence properties of the $\mathrm{Pr}^{3+}$ ions in the waveguide volume. A specific kind of guiding mode profiles is analyzed in detail, and simulation results suggest the feasibility of mode profile control in such waveguides. By means of the Raman property analysis of damage tracks and the surrounding areas, we have ascertained the modification effects of femtosecond laser and the refractive index induced guiding mechanism. With excellent luminescence performance and fairly low propagation losses, the fabricated waveguides present potential for integrated luminescence and laser generation applied in advances photonics circuits. 


\section{References}

1. Zhang D, Sugioka K. Hierarchical microstructures with high spatial frequency laser induced periodic surface structures possessing different orientations created by femtosecond laser ablation of silicon in liquids. Opto-Electron Adv 2, 190002 (2019).

2. Liu X, Bai B, Chen $Q$, Sun H. Etching-assisted femtosecond laser modification of hard materials. Opto-Electron Adv 2, 190021 (2019).

3. Zhang LM, Guo TY, Ren YY, Cai YJ, Mackenzie MD et al. Cooperative up-converted luminescence in $\mathrm{Yb}, \mathrm{Na}: \mathrm{CaF}_{2}$ cladding waveguides by femtosecond laser inscription. Opt Commun 441, 8-13 (2019).

4. Bharadwaj V, Courvoisier A, Fernandez TT, Ramponi R, Galzerano $G$ et al. Femtosecond laser inscription of Bragg grating waveguides in bulk diamond. Opt Lett 42, 3451-3453 (2017).

5. Sotillo B, Bharadwaj V, Hadden JP, Sakakura M, Chiappini A et al. Diamond photonics platform enabled by femtosecond laser writing. Sci Rep 6, 35566 (2016).

6. Li SL, Deng FM, Huang ZP. Femtosecond laser inscription waveguides in $\mathrm{Nd}: \mathrm{GdVO}_{4}$ crystal. Opt Eng 55, 107104 (2016).

7. Chandrahalim H, Rand SC, Fan XD. Fusion of renewable ring resonator lasers and ultrafast laser inscribed photonic waveguides. Sci Rep 6, 32668 (2016).

8. Vázquez MR, Sotillo B, Rampini S, Bharadwaj V, Gholipour B et al. Femtosecond laser inscription of nonlinear photonic circuits in Gallium Lanthanum Sulphide glass. J Phys Photonics 1, 015006 (2018).

9. Li SL, Ye YK, Shen CY, Wang HL. Femtosecond laser inscribed cladding waveguide structures in $\mathrm{LiNbO}_{3}$ crystal for beam splitters. Opt Eng 57, 117103 (2018).

10. Piromjitpong $\mathrm{T}$, Dubov $\mathrm{M}$, Boscolo $\mathrm{S}$. High-repetition-rate femtosecond-laser inscription of low-loss thermally stable waveguides in lithium niobate. Appl Phys A 125, 302 (2019).

11. Lv JM, Cheng YZ, Lu QM, Vázquez de Aldana JR, Hao XT et al. Femtosecond laser written optical waveguides in $z$-cut $\mathrm{MgO}: \mathrm{LiNbO}_{3}$ crystal: Fabrication and optical damage investigation. Opt Mater 57, 169-173 (2016).

12. Chen F, Vázquez de Aldana JRV. Optical waveguides in crystalline dielectric materials produced by femtosecond-laser micromachining. Laser Photonics Rev 8, 251-275 (2014).

13. Jia $\mathrm{Y}$, Wang $\mathrm{S}$, Chen F. Femtosecond laser direct writing of flexibly configured waveguide geometries in optical crystals: fabrication and application. Opto-Electron Adv 3, 190042 (2020).

14. Skryabin N, Kalinkin A, Dyakonov I, Kulik S. Femtosecond laser written depressed-cladding waveguide $2 \times 2,1 \times 2$ and $3 \times 3$ directional couplers in $\left.\mathrm{Tm}^{(3+}\right)$ :YAG crystal. Micromachines (Basel) 11, 1 (2019).

15. Ren YY, Jiao Y, Vázquez de Aldana JR, Chen F. Ti:Sapphire micro-structures by femtosecond laser inscription: Guiding and luminescence properties. Opt Mater 58, 61-66 (2016).

16. Bérubé JP, Lapointe J, Dupont A, Bernier M, Vallée R. Femtosecond laser inscription of depressed cladding single-mode mid-infrared waveguides in sapphire. Opt Lett 44, $37-40$ (2019).

17. Li SL, Huang ZP, Ye YK, Wang HL. Femtosecond laser in-

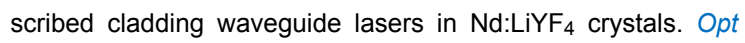
Laser Technol 102, 247-253 (2018).

18. Romero C, García Ajates J, Chen F, Vázquez de Aldana JR. Fabrication of tapered circular depressed-cladding wave- guides in Nd:YAG crystal by femtosecond-laser direct inscription. Micromachines (Basel) 11, 10 (2019).

19. Nie WJ, He RY, Cheng C, Rocha U, Vázquez de Aldana JR et al. Optical lattice-like cladding waveguides by direct laser writing: fabrication, luminescence, and lasing. Opt Lett 41, 2169-2172 (2016).

20. Liu HL, Luo SY, Xu B, Xu HY, Cai ZP et al. Femtosecond-laser micromachined Pr:YLF depressed cladding waveguide: Raman, fluorescence, and laser performance. Opt Mater Express 7, 3990-3997 (2017).

21. Okhrimchuk AG, Butvina LN, Dianov EM, Lichkova NV, Zagorodnev $\mathrm{VN}$ et al. New laser transition in a $\mathrm{Pr}^{3+}: \mathrm{RbPb}_{2} \mathrm{Cl}_{5}$ crystal in the 2.3-2.5- $\mu \mathrm{m}$ range. Quantum Electron 36, 41-44 (2006).

22. Nie HK, Zhang PX, Zhang BT, Yang KJ, Zhang LH et al. Diode-end-pumped Ho, Pr:LiLuF 4 bulk laser at $2.95 \mu \mathrm{m}$. Opt Lett 42, 699-702 (2017).

23. Fan MQ, Li T, Li GQ, Zhao SZ, Yang KJ et al. Passively Qswitched Ho, Pr:LiLuF 4 laser with graphitic carbon nitride nanosheet film. Opt Express 25, 12796-12803 (2017).

24. Cheng YJ, Peng J, Xu B, Yang H, Luo ZQ et al. Passive Qswitching of a diode-pumped Pr:LiYF 4 visible laser using $\mathrm{WS}_{2}$ as saturable absorber. IEEE Photonics J 8, 1-6 (2016).

25. Fibrich $M$, Šulc J, Zavadilová $A$, Jelínková $H$. Nonlinear mirror mode-locked Pr:YAIO 3 laser. Laser Phys 27, 055801 (2017).

26. Sattayaporn S, Loiseau P, Aka G, Marzahl DT, Kränkel C. Crystal growth, spectroscopy and laser performances of $\operatorname{Pr}\left({ }^{3+}\right): \mathrm{Sr}_{0.7} \mathrm{La}_{0.3} \mathrm{Mg}_{0.3} \mathrm{Al}_{11.7} \mathrm{O}_{19}$ (Pr:ASL). Opt Express 26, 1278-1289 (2018).

27. Wu PF, He S, Liu HL. Annular waveguide lasers at $1064 \mathrm{~nm}$ in $\mathrm{Nd}$ :YAG crystal produced by femtosecond laser inscription. Appl Opt 57, 5420-5424 (2018).

28. Liu HL, Vázquez de Aldana JR, Hong MH, Chen F. Femtosecond laser inscribed $\mathrm{Y}$-branch waveguide in Nd:YAG crystal: Fabrication and continuous-wave lasing. IEEE J Sel Top Quantum Electron 22, 227-230 (2016).

29. McDaniel S, Thorburn F, Lancaster A, Stites R, Cook G et al. Operation of Ho:YAG ultrafast laser inscribed waveguide lasers. Appl Opt 56, 3251-3256 (2017).

30. Kim MH, Calmano T, Choi SY, Lee BJ, Baek IH et al. Monolayer graphene coated Yb:YAG channel waveguides for Qswitched laser operation. Opt Mater Express 6, 2468-2474 (2016).

31. Gómez-Castellanos I, Rodriguez-Dagnino RM. Intensity distributions and cutoff frequencies of linearly polarized modes for a step-index elliptical optical fiber. Opt Eng 46, 045003 (2007).

\section{Acknowledgements}

We are grateful for financial supports from National Natural Science Foundation of China (NSFC) (Grants No. 61575097 and 11704201); National Natural Science Foundation of Tianjin City (NSFTJ) (17JCQNJC01600 and 19JCZDJC32700); the Fundamental Research Funds for the Central Universities.

\section{Author contributions}

H. L. Liu and P. F. Wu proposed the original idea and supervised the project. Q. X. Yang and S. He performed the experiments and measurements. Y. Q. Tian and B. Xu helped the luminescence characterization process.

\section{Competing interests}

The authors declare no competing financial interests. 